\title{
Prevalence of metabolic syndrome in patients with psoriasis: a cross-sectional study in Singapore
}

\author{
Wai Man Mandy $\underline{\text { Chan }}^{1}$, MBBS, MRCP, Yik Weng $\underline{\text { Yew }}{ }^{1}$, MBBS, MPH, Thiam Seng Colin Theng ${ }^{2}$, MBBS, MRCP,
} Choon Fong Liew $^{3}$, MBBS, MRCP, Hazel H OON${ }^{1}$, MD, MRCP

INTRODUCTION Psoriasis is a chronic inflammatory condition that affects the skin and joints, and is associated with cardiovascular risk factors, including metabolic syndrome (MetS). We aimed to assess the prevalence of MetS in patients with psoriasis and determine whether there was a correlation between psoriasis severity and MetS in a Singapore population.

METHODS This was a cross-sectional study of patients with psoriasis, aged 18-69 years, who attended a tertiary dermatology referral centre in Singapore from October 2007 to February 2009. Fasting glucose, lipids, blood pressure, Psoriasis Area and Severity Index, and body mass index were measured. MetS was diagnosed in the presence of three or more criteria of the modified National Cholesterol Education Program Adult Treatment Panel III.

RESULTS Among 338 patients with psoriasis, there were 238 (70.4\%) men and 100 (29.6\%) women, who were Chinese $(n=228 ; 67.5 \%)$, Malay $(n=52 ; 15.4 \%)$ and Indian $(n=58 ; 17.2 \%)$. The prevalence of MetS was $45.1 \%$. MetS was $44 \%$ more prevalent in patients older than 50 years $(p=0.02)$. Malay patients with psoriasis were significantly more likely to have hypertriglyceridaemia, elevated fasting plasma glucose and abdominal obesity. There was no significant correlation between psoriasis severity and risk of MetS.

CONCLUSION The prevalence of MetS in patients with psoriasis in Singapore was $45.1 \%$, or nearly threefold higher than the Singapore general population. Patients with psoriasis should be screened yearly for MetS and any modifiable cardiovascular risk factors should be actively controlled.

Keywords: metabolic syndrome, psoriasis, Singapore

\section{INTRODUCTION}

Psoriasis is a chronic inflammatory condition that affects approximately $1 \%-3 \%$ of the general population. ${ }^{(1)}$ Psoriasis is not only a disease of the skin and joints but also a systemic disease that is associated with a range of comorbidities, especially metabolic syndrome (MetS) and cardiovascular disease. Epidemiological studies suggest that psoriasis is associated with an increased frequency of cardiovascular risk factors and adverse cardiac outcomes, including myocardial infarction, stroke and cardiovascular death. ${ }^{(2-4)}$ MetS is a cluster of risk factors, including obesity, hypertension, dyslipidaemia and glucose intolerance, diagnosed according to proposed guidelines by various international groups. It is a strong predictor of cardiovascular disease, diabetes mellitus and stroke. ${ }^{(5)}$ The prevalence of MetS is increasing in parallel with the rising prevalence of obesity worldwide. ${ }^{(6)}$ It is important to recognise MetS early so that disease modification, such as weight loss and exercise, can target abdominal obesity and, more importantly, that cardiovascular risk factors, such as hypertension, diabetes mellitus and hyperlipidaemia, can be treated and reduced.

Both psoriasis and MetS share similarities, characterised by an inflammatory process driven by T-helper type 1 cytokines producing interferon and tumour necrosis factor- $\alpha .^{(7)}$ Increasingly, population-based studies have suggested a relationship between psoriasis and MetS. ${ }^{(8)}$ Patients with psoriasis have a greater prevalence of MetS as well as its individual components when compared to the general population. ${ }^{(9)}$ In Singapore, the prevalence of MetS in the community has been reported in the range of $12.2 \%-17.9 \%,{ }^{(10)}$ depending on the criteria adopted. MetS in Singapore has been associated with a threefold increase in cardiovascular mortality. ${ }^{(11)}$

The aim of our study was to examine MetS in patients with psoriasis in Singapore. Studies in the United Kingdom have also demonstrated that severity of psoriasis was associated with increasing risk of MetS. ${ }^{(12)}$ We wanted to examine whether a dose-response relationship was also observed between psoriasis severity and the prevalence of MetS in our Singapore population.

\section{METHODS}

This was a cross-sectional, tertiary centre-based study involving patients with psoriasis who attended a tertiary dermatology outpatient centre from October 2007 to February 2009. Inclusion criteria included patients in the age group 18-69 years and a clinical diagnosis of chronic plaque psoriasis. Written consent was obtained from each patient, and those who were unable to give consent and pregnant women were excluded from the study. The study was approved by our institutional review board.

Measurements, including anthropometric variables (e.g. weight, height, waist and hip circumference), systolic and diastolic blood pressure, fasting blood glucose and fasting lipid

${ }^{1}$ Department of Dermatology, National Skin Centre, ${ }^{2}$ The Skin Specialist and Laser Clinic, Mount Alvernia Medical Centre, ${ }^{3}$ Diabetes and Endocrine Centre, Raffles Medical Group, Singapore

Correspondence: Dr Hazel H Oon, Department of Dermatology, National Skin Centre, 1 Mandalay Road, Singapore 308205. hazeloon@nsc.com.sg 
profile, were taken. Weight and height were measured using a calibrated digital weighing scale and stadiometer, respectively. Waist circumference was measured with a circumference measurement tape. Waist was defined as the narrowest circumference between the iliac crest and the costal margin (lower rib), and hip was the widest circumference between the waist and thigh. Trained staff conducted all measurements with routine monitoring and quality checks. Body mass index was calculated based on the formula of weight in $\mathrm{kg} /$ height $^{2}$ in metres.

Blood pressure was measured using a standard mercury sphygmomanometer, taken on two separate visits with two readings on each visit. Non-diabetic patients were also subjected to a two-hour glucose challenge test. This involved a zero time (baseline) blood sample, after which patients were given a measured dose of $75 \mathrm{~g}$ of glucose solution to drink within five minutes, and blood samples were taken at one and two hours. Fasting glucose $<5.6 \mathrm{mmol} / \mathrm{L}$ was considered normal. Elevated fasting plasma glucose was defined as $\geq 5.6 \mathrm{mmol} / \mathrm{L}$. Impaired fasting glucose was defined as fasting plasma glucose in the range of 6.1-6.9 mmol/L. Levels above $11.1 \mathrm{mmol} / \mathrm{L}$ at two hours confirmed a diagnosis of diabetes mellitus.

MetS was diagnosed in the presence of three or more criteria of the National Cholesterol Education Program Adult Treatment Panel (NCEP-ATP) III guidelines (Table I). Serum cholesterol and triglycerides were measured using enzymatic procedures. Plasma glucose was measured using the glucose oxidase method.

A questionnaire on ethnicity, age, duration of psoriasis and self-reported diagnosis of diabetes mellitus, hypertension, hyperlipidaemia, ischaemic heart disease and lifestyle habits of smoking, alcohol consumption and physical exercise was also used. Patients who identified themselves as smokers were asked to document their daily cigarette intake and total pack year history. Patients who drank alcohol were asked whether they drank occasionally ( $<3$ drinks a month), frequently (1-4 drinks a week), regularly ( $>4$ drinks a week) or daily (to specify number of drinks daily). Patients who exercised were asked the type of exercise they did, number of sessions weekly and the duration (in minutes) of each session.

A dermatologist measured the Psoriasis Area and Severity Index (PASI) score. Patients with PASI $<10$ were considered to have mild-to-moderate psoriasis and those with PASI $\geq 10$ were considered to have severe psoriasis.

Data on patient demographics, clinical history of psoriasis, comorbid medical conditions, lifestyle factors, physical measurements and laboratory parameters were analysed and summarised using descriptive statistics.

To examine the relationship between severity of psoriasis and MetS, the risk of MetS was compared between patients of different psoriasis severity using chi-square test or Fisher's exact test. Kruskal-Wallis test was used to compare the differences in duration of psoriasis between different groups based on the components of MetS. Multivariate logistic regression analysis (robust Poisson regression models) for prevalence was performed with MetS being the dependent variable and age, diabetes mellitus, gender, ethnicity, current smoking status, alcohol
Table I. Modified NCEP-ATP III criteria.

\begin{tabular}{|ll|}
\hline Criteria & Modified NCEP-ATP III \\
\hline Obesity & \\
\hline Waist circumference $(\mathrm{cm})$ & $\geq 90$ \\
\hline Male & $\geq 80$ \\
\hline Female & $\geq 1.7^{*}$ \\
\hline Triglyceride (mmol/L) & $\leq 1.03^{*}$ \\
\hline HDL cholesterol (mmol/L) & $\leq 1.29^{*}$ \\
\hline Male & \\
\hline Female & $\geq 130^{\dagger}$ \\
\hline Blood pressure (mmHg) & $\geq 85^{\dagger}$ \\
\hline Systolic blood pressure & $\geq 5.6^{\ddagger}$ \\
\hline Diastolic blood pressure & \\
\hline Fasting plasma glucose (mmol/L) & \\
\hline
\end{tabular}

*Or drug treatment for elevated levels. †Or current use of antihypertensive drugs. $\neq$ Or self-reported diagnosis of diabetes mellitus. HDL: high-density lipoprotein; NCEP-ATP III: National Cholesterol Education Program Adult Treatment Panel III

consumption, exercise status and severity of psoriasis (PASI score) as independent variables. The statistical package STATA version 13 (STATA Corp, College station, TX, USA) was used for the analysis of the abovementioned variables to evaluate statistical significance. A p-value $<0.05$ was considered to be statistically significant.

\section{RESULTS}

A total of 338 patients, consisting of $238(70.4 \%)$ men and $100(29.6 \%)$ women, were enrolled in the study. Overall, $228(67.5 \%)$ patients were Chinese, $52(15.4 \%)$ patients were Malay and $58(17.2 \%)$ patients were Indian. The median age was 50.2 (range 19.4-69.7) years. Table II shows the baseline characteristics of patients with psoriasis in this study. Among our patients, 91 patients had pre-existing hypertension, with 53 patients on medication; 50 patients had known diabetes mellitus, with 37 patients on treatment; and 84 had known hyperlipidaemia, with 37 patients on medication.

The prevalence of MetS in patients with psoriasis was $45.1 \%$ (101/224 patients). The prevalence of each component of MetS was as follows: elevated blood pressure $(63.1 \%)$; abdominal obesity (60.1\%); low high-density lipoprotein cholesterol (35.6\%); hypertriglyceridaemia (29.7\%); and elevated fasting plasma glucose $(28.2 \%)$.

The risk of MetS based on severity of psoriasis, when analysed using chi-square test (Table III) or Fisher's exact test, suggested no association between the presence of MetS and the various components of MetS. Similarly, non-parametric Kruskal-Wallis tests also found no relationship between the duration of psoriasis, PASI score, presence of MetS and the various components of MetS (Table IV).

Yearly age was not a significant predictor of MetS after adjusting for other confounders upon multivariate logistic regression analysis. However, as we suspected that age may have had an impact on MetS, we used a median age of 50 years as a reference to classify patients into older (> 50 years) and younger ( $\leq 50$ years) groups. MetS was $44 \%$ more prevalent in patients with psoriasis aged above 50 years than patients aged below 
Table II. Baseline characteristics of study population $(n=338)$.

\begin{tabular}{|c|c|c|c|}
\hline Variable & No. (\%) & Variable & No. (\%) \\
\hline Age (yr) & & Mean \pm SD & $90.2 \pm 15.1$ \\
\hline Median (range) & $50.2(19.4-69.7)$ & Abdominal obesity $(n=223)$ & $134(60.1)$ \\
\hline Mean \pm SD & $48.7 \pm 11.4$ & Triglyceride $(n=219)$ & \\
\hline Age group (yr) & & Median (range) & $1.3(0.4-4.7)$ \\
\hline$\leq 50$ & $168(49.7)$ & Mean \pm SD & $1.5 \pm 0.9$ \\
\hline$>50$ & $170(50.3)$ & Hypertriglyceridaemia $(n=219)$ & $65(29.7)$ \\
\hline Gender & & HDL cholesterol $(n=219)$ & \\
\hline Male & $238(70.4)$ & Median (range) & $1.2(0.7-3.1)$ \\
\hline Female & $100(29.6)$ & Mean \pm SD & $1.2 \pm 0.3$ \\
\hline Ethnicity & & Low HDL cholesterol $(n=219)$ & $78(35.6)$ \\
\hline Chinese & $228(67.5)$ & LDL cholesterol $(n=219)$ & \\
\hline Malay & $52(15.4)$ & Median (range) & $3.2(0.8-5.9)$ \\
\hline Indian & $58(17.2)$ & Mean \pm SD & $3.3 \pm 0.8$ \\
\hline Systolic blood pressure $(n=223)$ & & Fasting plasma glucose $(n=220)$ & \\
\hline Median (range) & $131.0(93.0-189.0)$ & Median (range) & $4.9(3.7-22.2)$ \\
\hline Mean \pm SD & $132.5 \pm 17.8$ & Mean \pm SD & $5.6 \pm 2.1$ \\
\hline Diastolic blood pressure $(n=223)$ & & Elevated fasting plasma glucose & $62(28.2)$ \\
\hline Median (range) & $80.0(55.0-112.0)$ & $(n=220)$ & \\
\hline Mean \pm SD & $80.4 \pm 10.2$ & Diabetes mellitus ( $n=297$ ) & $50(16.8)$ \\
\hline Elevated blood pressure $(n=225)$ & $142(63.1)$ & Metabolic syndrome $(n=224)$ & $101(45.1)$ \\
\hline Body mass index $\left(\mathrm{kg} / \mathrm{m}^{2} ; \mathrm{n}=223\right)$ & & Duration of psoriasis $(y r ; n=296)$ & \\
\hline Median (range) & $25.7(16.3-47.6)$ & Median (range) & $15.0(0.1-50.0)$ \\
\hline Mean \pm SD & $26.1 \pm 5.1$ & Mean \pm SD & $14.9 \pm 9.8$ \\
\hline Body mass index $\left(\mathrm{kg} / \mathrm{m}^{2} ; \mathrm{n}=223\right)$ & & Psoriasis Area and Severity Index & \\
\hline$<23.0$ (healthy weight) & $62(27.8)$ & Median (range) & $4.2(0.0-56.1)$ \\
\hline 23.0-27.4 (overweight) & $84(37.7)$ & Mean \pm SD & $6.2 \pm 8.0$ \\
\hline$\geq 27.5$ (obese) & $77(34.5)$ & Smoking ( $n=297)$ & $136(45.8)$ \\
\hline Waist (cm; n = 223) & & Alcohol consumption $(n=297)$ & $129(43.4)$ \\
\hline Median (range) & $91.0(34.0-143.0)$ & Exercise $(n=297)$ & $170(57.2)$ \\
\hline
\end{tabular}

HDL: high-density lipoprotein; LDL: low-density lipoprotein; SD: standard deviation

50 years on adjusting for other baseline characteristics $(p=0.02)$ (Table V). Among patients with MetS, 44.9\% had PASI $<10$ and $46.3 \%$ of patients had PASI $\geq 10$ ( $p=0.84)$. No association was found between MetS and psoriasis severity after adjusting for other baseline characteristics.

Upon analysis for ethnic differences (Table VI), the adjusted prevalence of hypertriglyceridaemia in Malay patients with psoriasis was $81 \%$ higher than in Chinese patients $(p=0.04)$. The adjusted prevalence of low high-density lipoprotein cholesterol among Indian patients with psoriasis was $94 \%$ higher than in Chinese patients $(p<0.01)$. The adjusted prevalence of high fasting plasma glucose in Malay patients with psoriasis was 2.7 times higher than in Chinese patients $(p<0.01)$. The adjusted prevalence of abdominal obesity in Malay and Indian patients with psoriasis were $74 \%$ and $49 \%$ times higher, respectively, than in Chinese patients ( $p<0.01$ for both).

\section{DISCUSSION}

To our knowledge, this is the first and largest cross-sectional study of MetS among patients with psoriasis in Singapore. We found a marked increased prevalence of MetS in $45.1 \%$ of patients with psoriasis. There were no differences in the prevalence of MetS between men and women, but MetS was $44 \%$ more prevalent in patients with psoriasis aged above 50 years $(p=0.02)$. There was no significant correlation between psoriasis severity and risk of MetS in our population. Similar to the findings of the National Health Survey, abdominal obesity was higher among Indian and Malay patients with psoriasis compared to Chinese patients. ${ }^{(13)}$ Malay patients with psoriasis had higher triglyceride levels and elevated fasting plasma glucose levels, whereas Indian patients with psoriasis had lower high-density lipoprotein cholesterol when compared to Chinese patients. Early identification and subsequent referral of these subgroups to dietitians and nurses for lifestyle modifications and exercise may reduce their risk of developing cardiovascular disease.

Surprisingly, there were no significant associations found for MetS with smoking, alcohol consumption and exercise. This might be subject to recall and responder bias, where patients might have under-reported their smoking and alcohol risks while over-reporting their exercise habits.

The prevalence of MetS, in our study, was higher compared to the United Kingdom (34.2\%), ${ }^{(10)}$ Japan $(25.2 \%)$, ${ }^{(14)}$ Taiwan $(22.5 \%)^{(15)}$ and Italy $(30.1 \%) .{ }^{(16)}$ This could be possibly due to 
Table III. Association between psoriasis severity (PASI score) classification and components of metabolic syndrome.*

\begin{tabular}{|c|c|c|c|}
\hline \multirow[t]{2}{*}{ Variable } & \multicolumn{2}{|c|}{ PASI score classification (no. [\%]) } & \multirow[t]{2}{*}{ p-value } \\
\hline & $<10$ (mild-to-moderate psoriasis) $(n=157)$ & $\geq 10$ (severe psoriasis) $(n=67)$ & \\
\hline Metabolic syndrome & $156(100.0)$ & $67(100.0)$ & 0.848 \\
\hline No & $86(55.1)$ & 36 (53.7) & \\
\hline Yes & $70(44.9)$ & $31(46.3)$ & \\
\hline \multicolumn{4}{|l|}{ Components of metabolic syndrome } \\
\hline Elevated blood pressure & $157(100.0)$ & $67(100.0)$ & 0.803 \\
\hline No & $59(37.6)$ & $24(35.8)$ & \\
\hline Yes & $98(62.4)$ & $43(64.2)$ & \\
\hline Abdominal obesity & $155(100.0)$ & $67(100.0)$ & 0.466 \\
\hline No & $59(38.1)$ & $29(43.3)$ & \\
\hline Yes & $96(61.9)$ & $38(56.7)$ & \\
\hline Hypertriglyceridaemia & $152(100.0)$ & $66(100.0)$ & 0.588 \\
\hline No & $105(69.1)$ & $48(72.7)$ & \\
\hline Yes & $47(30.9)$ & $18(27.3)$ & \\
\hline Low high-density lipoprotein cholesterol & $152(100.0)$ & $66(100.0)$ & 0.463 \\
\hline No & $100(65.8)$ & $40(60.6)$ & \\
\hline Yes & $52(34.2)$ & $26(39.4)$ & \\
\hline Elevated fasting plasma glucose & $153(100.0)$ & $66(100.0)$ & 0.235 \\
\hline No & $114(74.5)$ & $44(66.7)$ & \\
\hline Yes & $39(25.5)$ & $22(33.3)$ & \\
\hline
\end{tabular}

*Based on the results of chi-square test. PASI: Psoriasis Area and Severity Index

Table IV. Non-parametric Kruskal Wallis tests for association between duration of psoriasis and the various components of metabolic syndrome.

\begin{tabular}{|c|c|c|c|}
\hline \multirow[t]{2}{*}{ Variable } & \multicolumn{2}{|c|}{ Duration of psoriasis (yr) } & \multirow[t]{2}{*}{ p-value* } \\
\hline & Median (range) & Mean \pm SD & \\
\hline \multicolumn{3}{|c|}{ Metabolic syndrome } & 0.864 \\
\hline No & $15.0(0.5-40.0)$ & $15.0 \pm 9.5$ & \\
\hline Yes & $15.0(0.1-38.0)$ & $15.0 \pm 10.1$ & \\
\hline \multicolumn{4}{|c|}{ Components of metabolic syndrome } \\
\hline \multicolumn{3}{|c|}{ Elevated blood pressure } & 0.296 \\
\hline No & $13.5(0.5-37.0)$ & $13.9 \pm 9.2$ & \\
\hline Yes & $15.0(0.1-40.0)$ & $15.6 \pm 10.0$ & \\
\hline \multicolumn{3}{|c|}{ Abdominal obesity } & 0.314 \\
\hline No & $15.0(0.1-38.0)$ & $15.7 \pm 9.7$ & \\
\hline Yes & $15.0(0.3-40.0)$ & $14.5 \pm 9.8$ & \\
\hline \multicolumn{3}{|c|}{ Hypertriglyceridaemia } & 0.263 \\
\hline No & $15.0(0.1-40.0)$ & $14.4 \pm 9.4$ & \\
\hline Yes & $17.0(0.1-38.0)$ & $16.2 \pm 10.5$ & \\
\hline \multicolumn{3}{|c|}{ Low high-density lipoprotein cholesterol } & 0.088 \\
\hline No & $15.0(0.1-40.0)$ & $15.7 \pm 9.6$ & \\
\hline Yes & $11.5(0.3-37.0)$ & $13.6 \pm 9.9$ & \\
\hline \multicolumn{3}{|c|}{ Elevated fasting plasma glucose } & 0.371 \\
\hline No & $15.0(0.3-40.0)$ & $14.4 \pm 9.0$ & \\
\hline Yes & $15.0(0.1-38.0)$ & $16.4 \pm 11.3$ & \\
\hline
\end{tabular}

*For difference in PASI score between groups. PASI: Psoriasis Area and Severity Index; SD: standard deviation
Table V. Prevalence of metabolic syndrome in patients with psoriasis after adjusting for psoriasis severity and other baseline characteristics.

\begin{tabular}{|lll|}
\hline Variable & $\begin{array}{c}\text { Adjusted prevalence } \\
\text { ratio (95\% CI) }\end{array}$ & p-value \\
\hline Age (yr) & \\
\hline$\leq 50$ & $1.44(1.06-1.96)$ & $0.02^{*}$ \\
\hline$>50$ & \\
\hline Gender & \\
\hline Male (ref) & $1.28(0.89-1.85)$ & 0.18 \\
\hline Female & \\
\hline Ethnicity & \\
\hline Chinese (ref) & $1.43(0.94-2.16)$ & 0.09 \\
\hline Malay & $1.26(0.92-1.73)$ & 0.15 \\
\hline Indian & \\
\hline PASI score & & \\
\hline$<10$ (ref) & $1.18(0.88-1.58)$ & 0.28 \\
\hline$\geq 10$ & $0.99(0.97-1.00)$ & 0.13 \\
\hline Duration of psoriasis (yr) & $1.14(0.77-1.67)$ \\
\hline Smoking & $0.98(0.69-1.39)$ & 0.51 \\
\hline Alcohol consumption & $1.15(0.86-1.54)$ & 0.91 \\
\hline Exercise & $2.11(1.61-2.79)$ & $<0.35$ \\
\hline Diabetes mellitus & & \\
\hline
\end{tabular}

${ }^{*} \mathrm{p}<0.05$ was statistically significant. Cl: confidence interval; PASI: Psoriasis Area and Severity Index 
Table VI. Ethnic differences for each component of metabolic syndrome.*

\begin{tabular}{|lll|}
\hline Variable & $\begin{array}{c}\text { Adjusted prevalence } \\
\text { ratio }(95 \% \text { Cl) }\end{array}$ & p-value \\
\hline Hypertriglyceridaemia & \\
\hline Malay & $1.81(1.03-3.19)$ & $0.04^{\dagger}$ \\
\hline Indian & $0.94(0.52-1.69)$ & 0.83 \\
\hline \multicolumn{2}{|l|}{ Low high-density lipoprotein cholesterol } \\
\hline Malay & $0.88(0.49-1.60)$ & 0.69 \\
\hline Indian & $1.94(1.31-2.88)$ & $<0.01^{\dagger}$ \\
\hline Elevated fasting plasma glucose & \\
\hline Malay & $2.71(1.52-4.82)$ & $<0.01^{\dagger}$ \\
\hline Indian & $1.54(0.94-2.53)$ & 0.09 \\
\hline Raised blood pressure & $1.27(0.91-1.78)$ \\
\hline Malay & $0.80(0.60-1.07)$ & 0.17 \\
\hline Indian & 0.13 \\
\hline Abdominal obesity & $1.74(1.37-2.21)$ \\
\hline Malay & $1.49(1.17-1.91)$ \\
\hline Indian & $<0.01^{\dagger}$ \\
\hline
\end{tabular}

*Patients of Chinese ethnicity were taken as reference. $t p<0.05$ was statistically significant. $\mathrm{Cl}$ : confidence interval

the varying criteria used for diagnosing MetS among the various populations. Various organisations have proposed criteria for diagnosing MetS, including the NCEP-ATP III, (17) International Diabetes Foundation, World Health Organization and European Group for the Study of Insulin Resistance. For Asians, a lower waist circumference cut-off point for men and women is represented in the modified NCEP-ATP III guidelines, and this was the defining criterion used in our study.

The waist circumference in the modified NCEP-ATP III criteria, when compared to the NCEP-ATP III, is lower for men (NCEP-ATP III: $\geq 102 \mathrm{~cm}$; modified NCEP-ATP III: $\geq 90 \mathrm{~cm}$ ) and women (NCEP-ATP III: $\geq 88 \mathrm{~cm}$; modified NCEP-ATP III: $\geq 80 \mathrm{~cm}$ ). Using the original NCEP-ATP III criteria, the prevalence of MetS would be $33 \%$, compared with $45.1 \%$ when using the modified criteria for our cohort. This may be explained by an increase in the prevalence of abdominal obesity in our cohort, with $60.1 \%$ of patients having abdominal obesity when compared to $16.9 \%$ of the general population in the National Health Survey conducted in Singapore in 2010. ${ }^{(18)}$ The increase in abdominal obesity noticed in our patients also reflected an increase in the prevalence of MetS consequent to the use of the modified NCEP-ATP III criteria. On similar lines, the prevalence of MetS among patients with psoriasis in North India ${ }^{(19)}$ was $42 \%$ using the modified NCEP-ATP III criteria. In view of the rising trend of abdominal obesity in Asia, these findings suggest that perhaps the NCEP-ATP III criteria should be used instead of the modified criteria to prevent any unintended 'inflation' of the prevalence of MetS.

Our study had its strengths and weaknesses. One of its strengths was the multiethnicity of our Singapore cohort, allowing us to make comparisons to evaluate if ethnicity influenced the risk of MetS among patients with psoriasis. Our study had a large sample size and robust measurement methods for detecting
MetS instead of only relying on self-reporting or diagnosis codes. Moreover, the severity of psoriasis was measured objectively by a clinician based on PASI scoring.

Limitations of this study were that it was conducted at a tertiary-care centre and so our cohort might not have been a good representation of the psoriasis demographics in the community. Our results may be more skewed, as patients approaching for tertiary care tend to have more severe psoriasis and a longer duration of disease when compared to those in the community. There was also no control group and data was compared to findings of the National Health Survey. ${ }^{(18)}$ Notably, there were significantly more men $(70.4 \%)$ compared to women $(29.6 \%)$ in our study. Given the cross-sectional nature of the study, our results might be subjected to reverse causation bias and therefore we were unable to infer causality between psoriasis and MetS. Among the original cohort of 338 patients, there was missing data for up to $35.2 \%$ ( $n=119$ ) of patients, including lipid levels, body mass index, blood pressure and abdominal obesity. Data was missing for patients, as it had not been recorded in the electronic medical record system or because the relevant laboratory tests were not done during follow-up. Results were still analysed and patients were followed up. Other factors, including diet or family history, could have played a role, but such information was not readily available in our electronic medical records.

In our clinical practice, our tertiary outpatient centre has implemented an automated annual pop-up screen ${ }^{(20)}$ for all patients diagnosed with psoriasis to remind attending physicians to screen these patients yearly for MetS. Patient education and outreach programmes emphasising the relationship between MetS, psoriasis and cardiovascular risk factors have also improved patient knowledge and understanding. Another limitation of this study was that the sample population might not have been representative of the general population. Larger-scale populationbased studies may be undertaken to determine the underlying association between these two variables, and to monitor the effects and outcome of early intervention and management of MetS.

In conclusion, our study demonstrated a high prevalence of MetS in patients with psoriasis in Singapore, and emphasised the need for vigorous screening for components of MetS. However, we did not observe any correlation between disease severity and presence of MetS. Abdominal obesity seemed to play an integral role in an increased prevalence of MetS, and physicians need to be aware of the rising trend of abdominal obesity in Asia. Focused therapies can be employed to target abdominal obesity, especially among Malay and Indian patients, such as referral to physiotherapists and sport trainers for tailored exercise programmes, and early referrals to dietitians to improve low levels of low-density lipoprotein cholesterol in Indian patients and elevated fasting plasma glucose levels in Malay patients. Early diagnosis of MetS will allow for intensive risk factor modification, including lifestyle interventions, weight loss, treatment of hypertension, diabetes mellitus and hyperlipidaemia, to reduce the risk of cardiovascular disease and mortality in patients with psoriasis. 


\section{ACKNOWLEDGEMENTS}

We acknowledge and thank Ms Virlynn Tan, Biostatistician, Research Department, National Skin Centre, Singapore, for her statistical input. The study was funded by the National Medical Research Council enabling grant. The funders had no involvement in the study design, data collection, analysis, interpretation and writing of the manuscript. The authors do not have further declarations regarding funding that supported the work. Dr Hazel $\mathrm{H}$ Oon has served as a speaker and advisory board member for AbbVie and Galderma. She is a clinical investigator for Janssen, Novartis and Pfizer. This did not have any conflict of interest with the preparation of this manuscript. The authors have no further conflicts of interest to declare.

\section{REFERENCES}

1. Gelfand JM, Weinstein R, Porter SB, et al. Prevalence and treatment of psoriasis in the United Kingdom: a population-based study. Arch Dermatol 2005 141:1537-41.

2. Gelfand JM, Neimann AL, Shin DB, et al. Risk of myocardial infarction in patients with psoriasis. JAMA 2006; 296:1735-41.

3. Gelfand JM, Dommasch ED, Shin DB, et al. The risk of stroke in patients with psoriasis. J Invest Dermatol 2009; 129:2411-8.

4. Gelfand JM, Azfar RS, Mehta NN. Psoriasis and cardiovascular risk: strength in numbers. J Invest Dermatol 2010; 130:919-22.

5. Malhotra SK, Dhaliwal GS, Puri KJPS, Gambhir ML, Mahajan M. An insight into relationship between psoriasis and metabolic syndrome. Egypt Dermatol Online J 2011; 7:5.

6. Ford ES, Giles $\mathrm{WH}$, Mokdad $\mathrm{AH}$. Increasing prevalence of the metabolic syndrome among US adults. Diabetes Care 2004; 27:2444-9.

7. Sterry W, Strober BE, Menter A; International Psoriasis Council. Obesity in psoriasis: the metabolic, clinical and therapeutic implications. Report of an interdisciplinary conference and review. Br J Dermatol 2007; 157:649-55.
8. Armstrong AW, Harskamp CT, Armstrong EJ. Psoriasis and metabolic syndrome: a systematic review and meta-analysis of observational studies. J Am Acad Dermatol 2013; 68:654-62.

9. Singh S, Young P, Armstrong AW. Relationship between psoriasis and metabolic syndrome: a systematic review. G Ital Dermatol Venereol 2016; 151:663-77.

10. Tan CE, Ma S, Wai D, Chew SK, Tai ES. Can we apply the National Cholesterol Education Program Adult Treatment Panel definition of the metabolic syndrome to Asians? Diabetes Care 2004; 27:1182-6.

11. Lee J, Heng D, Ma S, et al. The metabolic syndrome and mortality: the Singapore Cardiovascular Cohort Study. Clin Endocrinol (Oxf) 2008; 69:225-30.

12. Langan SM, Seminara NM, Shin DB, et al. Prevalence of metabolic syndrome in patients with psoriasis: a population-based study in the United Kingdom. J Invest Dermatol 2012; 132(3 Pt 1):556-62.

13. Goh LG, Pang J. Obesity in Singapore, prevention and control. Singapore Fam Physician 2012; 38:8-13.

14. Takahashi H, Takahashi I, Honma M, Ishida-Yamamoto A, lizuka H. Prevalence of metabolic syndrome in Japanese psoriasis patients. J Dermatol Sci 2010; 57:143-4.

15. Chen YJ, Wu CY, Shen JL, et al. Psoriasis independently associated with hyperleptinemia contributing to metabolic syndrome. Arch Dermatol 2008; 144:1571-5

16. Gisondi P, Tessari G, Conti A, et al. Prevalence of metabolic syndrome in patients with psoriasis: a hospital-based case-control study. Br J Dermatol 2007; 157:68-73.

17. Expert Panel on Detection, Evaluation, and Treatment of High Blood Cholesterol in Adults. Executive summary of the third report of the National Cholesterol Education Program (NCEP) expert panel on detection, evaluation, and treatment of high blood cholesterol in adults (adult treatment panel III). JAMA 2001; 285:2486-97.

18. Epidemiology and Disease Control Division, Ministry of Health, Singapore. National Health Survey 2010. Available at: https://www.moh.gov.sg/content/ dam/moh_web/Publications/Reports/2011/NHS2010\%20-\%20low\%20res.pdf. Accessed July 18, 2017

19. Dalal A, Mahendra A, Gupta S. A study of prevalence of metabolic syndrome in patients of psoriasis of North India. Br J Med Med Res 2016; 11:1-8.

20. Wang EC, Ker KJ, Chuah SY, Pan JY. Obstacles and solutions to screening psoriasis patients for cardiovascular risk factors. J Eur Acad Dermatol Venereol 2014; 28:819-23. 